\title{
Association between Maternal Adverse Childhood Experiences and Risk of Post-traumatic Stress Disorder in the Offspring
}

\author{
Min Chull Na', Moon Doo Kim ${ }^{1,2}$, Joon Hyuk Park ${ }^{1,2}$, Young-Eun Jung, ${ }^{1,2}$, \\ Duk-Soo Moon', Hyun-Ju Yang', Bung-Nyun $\mathrm{Kim}^{3}$, and Na Ri Kang ${ }^{1,2}$ \\ ${ }^{1}$ Department of Psychiatry, Jeju National University Hospital, Jeju, Korea \\ ${ }^{2}$ Department of Psychiatry, Jeju National University School of Medicine, Jeju, Korea \\ ${ }^{3}$ Department of Psychiatry, Seoul National University School of Medicine, Seoul, Korea
}

\begin{abstract}
Objectives: Adverse childhood experiences (ACEs) of mothers may negatively affect the mental health of their offspring. Little is known about the intergenerational effect of maternal ACE on post-traumatic stress disorder (PTSD) in the offspring. This study investigated the impact of maternal ACEs on PTSD in the offspring.

Methods: A total of 156 mothers with children aged 13-18 years completed the Diagnostic Interview Schedule for Children (DISC) Predictive Scales to determine the presence of psychiatric disorders in their offspring. The subjects completed the ACE questionnaire and the Early Trauma Inventory Self-Report-Short Form. Multivariable logistic regression was used to analyze the relationship between maternal ACEs and PTSD in the offspring.

Results: Of the mothers, $23.7 \%$ had at least one ACE, and PTSD was reported in $21.8 \%$ of the offspring. The offspring of the mothers in the ACE group had a significantly higher rates of traumatic experiences and PTSD than the offspring of the mothers in the no ACE group. Maternal household dysfunction independently predicted offspring PTSD [odds ratio $(\mathrm{OR})=3.008, \mathrm{p}=0.05)$, and three or more maternal ACEs were significantly related to PTSD in the offspring ( $\mathrm{OR}=10.613, \mathrm{p}=0.025)$.

Conclusion: Maternal ACEs have a significant impact on the risk of traumatic experiences and PTSD in the offspring. These findings suggest the presence of intergenerational transmissions by which maternal ACEs affect the mental health of the offspring.
\end{abstract}

Key Words: Maternal; Adverse childhood experiences; Offspring; Post-traumatic stress disorder.

Received: December 10, 2020 / Revision: January 6, 2021 / Accepted: January 19, 2021

Address for correspondence: Na Ri Kang, Department of Psychiatry, Jeju National University School of Medicine, 15 Aran 13-gil, Jeju 63241, Korea Tel: +82-64-717-1850, Fax: +82-64-717-1849, E-mail: ilnaree@hanmail.net

\section{INTRODUCTION}

Adverse childhood experiences (ACEs) are those experiences in which individuals aged below 18 years are exposed to traumatic events such as child abuse, neglect, and household dysfunction [1]. Many studies have found that the risks of adverse health behaviors, mental health problems, and chronic disease over the lifetime increase in proportion to the ACEs. In particular, exposure to ACEs is three times higher in persons experiencing depressive symptoms than in non-depressed persons; persons with mental illness, including mood disorder, anxiety, substance abuse, and psychosis have usually had more than three ACEs [2]. A previous study on ACEs and psychiatric disorders in young adulthood reported that child

This is an Open Access article distributed under the terms of the Creative Commons Attribution Non-Commercial License (https://creativecommons.org/licenses/by-nc/4.0) which permits unrestricted non-commercial use, distribution, and reproduction in any medium, provided the original work is properly cited. maltreatment experience leads to a 1.97 -fold higher risk of post-traumatic stress disorder (PTSD) in adulthood [3].

Recently, evidence has shown that maternal ACEs are associated with offspring developmental delay, behavioral problems, social-emotional functioning, and psychopathology through intergenerational transmission [4]. Studies have shown that maternal trauma history is directly associated with anxiety in their offspring, and maternal experience of childhood abuse is associated with depressive symptoms in the offspring during adolescence [5]. In addition, it has been reported that children of parents with a history of four or more ACEs have, on average, 4.2-fold higher odds of emotional disturbance than children of parents with no ACEs [6]. These results show the cumulative dose-response-based intergenerational transmission of maternal ACEs.

ACE, namely parental exposure to childhood maltreatment, is an important predictor of maltreatment experiences and 
mental health problems in the offspring. In particular, considering that maltreatment of the parents in their childhood is significantly related to PTSD symptoms in their offspring [7], maternal ACEs are a risk factor for PTSD not only in the mothers but also in their offspring. However, the association between maternal ACEs and PTSD in the offspring has not yet been examined. We hypothesized that when maternal ACEs are present, the risk of traumatic experience and the frequency of PTSD for the offspring is high, and maternal ACE is a significant risk factor for PTSD in the offspring. The present study evaluated the prevalence of maternal ACEs and the prevalence of traumatic experiences and PTSD in the offspring. We also examined the association between maternal ACEs, including child abuse, neglect, and household dysfunction, and PTSD in the offspring and the effect of cumulative maternal ACEs.

\section{METHODS}

\section{Participants and procedure}

The participants included mothers with children aged 13 years to 18 years who had attended school for more than 6 months in Jeju Special Self-Governing Province, Korea. Two middle schools and high schools were selected with the cooperation of the Jeju Special Self-Governing Province Office of Education. We sent the survey forms, including the study background and consent to participate, to parents of students in all grades. For data collection, the survey questionnaires were distributed and collected by trained mental health specialists. A total of 245 mother-child dyads participated in this study. Among them, 156 mother-child dyads, excluding those with psychiatric disorders other than PTSD, participated in the final study (Fig. 1). The data were collected from September to December 2017. Written consent was obtained from the mothers and their children. This study was approved by the Institutional Review Board of the Jeju National University Hospital (Grant No. 2018-08-004).

\section{Assessments}

\section{Adverse childhood experiences questionnaire}

The ACE questionnaire collects retrospective data about childhood experiences until the age of 18. It includes 10 ACEs for child abuse (e.g., verbal, physical, and sexual abuse), child neglect (e.g., emotional and physical neglect) and household dysfunction (e.g., parental divorce, domestic violence, alcohol problem, mental illness, and prison) [8]. When the response to the ACE item is "yes," it is assigned a score of one point; the accumulated points are added to obtain the total ACE score. In this study, we analyzed the relationship between the total

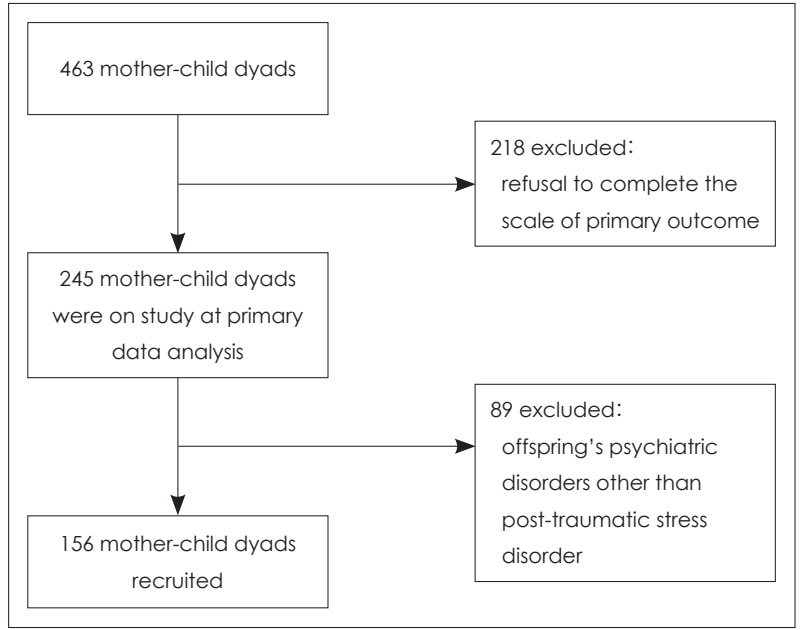

Fig. 1. Recruitment profile.

ACE score, three domains (child abuse, child neglect, and household dysfunction) of ACE, and PTSD in the offspring.

\section{Early Trauma Inventory Self-Report-Short Form}

The Early Trauma Inventory Self-Report-Short Form (ETISRSF) consists of 27 items about traumatic experiences that may have occurred until the age of 18 in four domains: general (11 items), physical (5 items), emotional (5 items), and sexual trauma (6 items) [9]. Each traumatic experience was scored dichotomousely (yes $=1, \mathrm{no}=0$ ). We used the Korean version of the ETISR-SF [10], which is a reliable and valid instrument for measuring childhood traumatic experiences. The total ETISRSF scores and scores for each of the four domains were used for analysis, with each trauma experience assigned one point.

\section{Diagnostic Interview Schedule for Children Predictive Scales}

The Predictive Scales (DPS) includes only the Diagnostic Interview Schedule for Children (DISC) items that are most predictive of a Diagnostic and Statistical Manual of Mental Disorders, fourth edition (DSM-IV) diagnosis [11] and evaluates the psychiatric symptoms the children display in the last year. DPS has been widely examined, showing high test-retest reliability and internal consistency [12]. A previous epidemiological survey of children and adolescents conducted in South Korea [13] included 33 questions about diagnoses previously found in the DISC-IV but not in the DPS (selective mutism, eating disorder, elimination disorder, PTSD, tic disorder, nicotine dependence, pica, and trichotillomania). The present study used the modified DPS; the DPS of offspring's psychiatric disorders was completed by the mothers. Each psychiatric disorder's diagnostic cutoff was defined according to Cubo et al. [14]. This study analyzed PTSD groups diagnosed according to the PTSD cutoff score and used a control group 
that included offspring without psychiatric disorders, excluding psychiatric disorders other than PTSD.

\section{Center for Epidemiological Studies Depression Scale for Children}

The Center for Epidemiological Studies Depression Scale for Children (CES-DC), which is developed by the National Institute of Mental Health (NIMH), is a scale used to examine the degrees of depressive symptoms in children and adolescents aged 6-17 years [15]. We used the standardized CESDC [16]; a high CES-DC score indicates the presence of severe depressive.

\section{Screen for Children Anxiety-Related Disorders}

To measure adolescent anxiety, we used the Screen for Children Anxiety-Related Disorders (SCARED), which was developed by Birmaher et al. [17] and revised by Kim [18]. It is a self-report scale consisting of 41 questions. The items are scored from 0 to 2, representing not at all, sometimes, and often, respectively. A higher score indicates a higher level of anxiety.

\section{Beck Depression Inventory-II}

The Beck Depression Inventory (BDI-II), a self-report scale developed by Beck et al. [19], is based on the criteria for depressive disorder in the DSM-IV and consists of 21 questions on cognitive, emotional, and physiological symptoms of depression. The items are scored on a Likert scale from 0 point to 3 points, and the scores for the 21 items are summed; the total score ranges from 0 point to 62 points, where the higher the score the more severe the depression. In the present study, the BDI-II was used to evaluate the severity of maternal depression; we used the standardized scale for the Korean population (Cronbach's $\alpha=0.91$ ) [20].

The mothers completed the ACE questionnaire about maternal ACEs. They also filled in the ETISR-SF and DPS on offspring's traumatic experience and psychiatric disorders. The offspring answered the CES-DC and SCARED on depression and anxiety, respectively. The CES-DC, SCARED, and maternal BDI-II scores were used in the regression analysis as variables that can affect PTSD in the offspring.

\section{Statistical analysis}

We analyzed the participants' sociodemographic characteristics, frequency of ACE domains, and offspring's traumatic experience through descriptive analysis. The frequency of the offspring's traumatic experience and PTSD, according to maternal ACEs, was verified by the chi-squared test; the sum of traumatic experiences was tested by the independent sample t-test. The chi-squared analysis was carried out to compare the frequency of a maternal ACE item between the PTSD group and the control group. A multivariate logistic regression analysis was conducted to adjust for the emotional state of the children and mothers using CES-DC, SCARED, maternal BDI-II, and socioeconomic variables such as socioeconomic status, maternal age, maternal education level, and offspring's age and sex. We examined the odds ratios (OR) and 95\% confidence intervals (CI) of the total maternal ACE score and the scores on the three domains of maternal ACEs in predicting PTSD in the offspring. SPSS version 18 (SPSS Inc., Chicago, IL, USA) was used for data analysis, and the significance level of all statistical analyses was $\mathrm{p}<0.05$.

\section{RESULTS}

\section{Sociodemographic characteristics of the participants}

Of the total 156 mother-child dyads, the mean age of the subjects was $45.74( \pm 4.49)$ years and the mean age of the offspring was $16.58( \pm 1.47)$ years; the offspring groups consisted of $62.8 \%(n=98)$ females and $37.2 \%(n=58)$ males. A total of $51.0 \%(\mathrm{n}=75)$ of mothers were in the middle socioeconomic status, and $96.7 \%(\mathrm{n}=143)$ had education level of high school graduate or higher.

\section{Maternal ACEs and offspring's traumatic experiences}

In total, $23.7 \%(\mathrm{n}=37)$ mothers had more than one ACE, and $7.7 \%(\mathrm{n}=12)$ had more than three ACEs. When classified into three domains, $8.3 \%(\mathrm{n}=13)$ experienced child abuse, 5.1\% $(\mathrm{n}=8)$ experienced child neglect, and $21.2 \%(\mathrm{n}=32)$ experienced household dysfunction, which was the most frequently reported ACE. Regarding the offspring's traumatic experiences, $28.2 \%(n=44)$ experienced general trauma, while $18.6 \%(n=$ 29), $10.9 \%(\mathrm{n}=17)$, and $0.6 \%(\mathrm{n}=1)$ experienced physical, emotional, and sexual trauma, respectively. In total, $35.9 \%(n=56)$ of the offspring had more than one traumatic experience and $12.8 \%(n=20)$ had more than three traumatic experiences (Table 1). A total of $21.8 \%$ ( $n=34$ ) of the offspring were in the PTSD group (Table 1), of which $47.1 \%$ ( $n=16$ ) had only PTSD and $52.9 \%$ had PTSD with comorbid disorders. The most common comorbid disorders were attention-deficit/hyperactivity disorder, anxiety disorder, and major depressive disorder.

\section{Frequency of offspring's traumatic experiences and PTSD according to maternal ACEs}

There was no significant difference between the demographic variables and the offspring's CES-DC and SCARED scores according to maternal ACEs. Maternal BDI-II score was significantly higher in the group with maternal ACEs than in group without maternal ACEs (Table 2). The prevalence rates of gen$\operatorname{eral}(56.8 \%$ vs. $19.3 \%$; $\mathrm{p}<0.001)$, physical $(43.2 \%$ vs. $10.9 \%$; $\mathrm{p}<$ 
$0.001)$, and emotional traumas $(24.3 \%$ vs. $6.7 \%$; $\mathrm{p}=0.003)$ in the offspring were significantly higher in the group of mothers with ACEs than in the group without. The total number

Table 1. Frequency of maternal ACEs and offspring's traumatic experiences

\begin{tabular}{|c|c|}
\hline & Values $(n=156)$ \\
\hline \multicolumn{2}{|l|}{ Maternal ACEs } \\
\hline Child abuse, $\geq 1$ & $13(8.3)$ \\
\hline Child neglect, $\geq 1$ & $8(5.1)$ \\
\hline Household dysfunction, $\geq 1$ & $33(21.2)$ \\
\hline \multicolumn{2}{|l|}{ Total ACE score } \\
\hline 0 & $119(76.3)$ \\
\hline 1 & $18(11.5)$ \\
\hline 2 & $7(4.5)$ \\
\hline$\geq 3$ & $12(7.7)$ \\
\hline \multicolumn{2}{|l|}{ Offspring's ETISR-SF } \\
\hline General trauma, $\geq 1$ & $44(28.2)$ \\
\hline Physical trauma, $\geq 1$ & $29(18.6)$ \\
\hline Emotional trauma, $\geq 1$ & $17(10.9)$ \\
\hline Sexual trauma, $\geq 1$ & $1(0.6)$ \\
\hline \multicolumn{2}{|l|}{ Total ETISR-SF score } \\
\hline 0 & $100(64.1)$ \\
\hline 1 & $22(14.1)$ \\
\hline 2 & $14(9.0)$ \\
\hline$\geq 3$ & $20(12.8)$ \\
\hline Offspring's PTSD & $34(21.8)$ \\
\hline
\end{tabular}

Data are presented as $n$ (\%). ACE: adverse childhood experience, ETISR-SF: Early Trauma Inventory Self-Report-Short Form, PTSD: post-traumatic stress disorder of traumatic experiences of the offspring was $2.78( \pm 3.63)$ for those with maternal ACEs, significantly higher than that for the group without maternal ACEs $(0.55 \pm 1.30, \mathrm{p}<0.001)$. The frequency of PTSD in the offspring was $51.4 \%(n=19)$, significantly higher in the group with maternal ACEs than in the group without $[12.6 \%(n=15)]$ (Table 3$)$.

\section{PTSD in the offspring and maternal ACEs}

Verbal abuse (20.6\% vs. $1.6 \%$ ), physical abuse (17.6\% vs. $1.6 \%$ ), emotional neglect (17.6\% vs. $1.6 \%)$, divorce ( $32.4 \%$ vs. $4.9 \%)$, domestic violence ( $14.7 \%$ vs. $5.7 \%)$, alcohol problems $(20.6 \%$ vs. $5.7 \%$ ), and mental illness ( $17.6 \%$ vs. $2.5 \%$ ) of family members, excluding sexual abuse, physical neglect, and prison sentence, were significantly higher in the PTSD group than in the control group (Fig. 2). When the mother experienced household dysfunction among the three domains of ACEs, the OR of their offspring falling under the PTSD group was 3.008 times higher than those with mothers without household dysfunction and statistically significant $\left(\mathrm{OR}=3.008,95 \% \mathrm{CI}=1.238^{-}\right.$ 7.305, $\mathrm{p}=0.05$ ) (Table 4). The OR was significantly higher when the ACE score was 3 or more $\left(\mathrm{OR}=10.613,95 \% \mathrm{CI}=1.338^{-}\right.$ $84.172, \mathrm{p}=0.025$ ) than when ACE was 2 or below (Table 5).

\section{DISCUSSION}

The frequencies of traumatic experiences and PTSD were significantly higher in the offspring whose mothers had ACEs than in the offspring whose mothers did not. Among ACEs, household dysfunction was significantly associated with PTSD,

Table 2. Comparison of demographic variables according to maternal ACEs

\begin{tabular}{|c|c|c|c|}
\hline & Maternal ACEs $(+)(n=37)$ & Maternal ACEs (-) $(n=119)$ & $\mathrm{p}$ \\
\hline \multicolumn{4}{|l|}{ Children related variables } \\
\hline Children's sex (male) & $12(32.4)$ & $46(38.7)$ & 0.494 \\
\hline Children's age, years & $16.41 \pm 1.54$ & $16.63 \pm 1.45$ & 0.418 \\
\hline CES-DC & $15.06 \pm 11.97$ & $13.89 \pm 9.11$ & 0.540 \\
\hline SCARED & $17.17 \pm 15.02$ & $14.10 \pm 10.99$ & 0.190 \\
\hline \multicolumn{4}{|l|}{ Mother related variables } \\
\hline Maternal age, years & $46.27 \pm 5.80$ & $45.58 \pm 4.01$ & 0.440 \\
\hline Maternal education level & & & 0.206 \\
\hline High ( > high school) & $18(52.9)$ & $41(36.0)$ & \\
\hline Middle (high school) & $15(44.1)$ & $69(60.5)$ & \\
\hline Low $(\leq$ middle school) & $1(2.9)$ & $4(3.5)$ & \\
\hline Socioeconomic status & & & 0.110 \\
\hline High & $6(16.7)$ & $15(13.5)$ & \\
\hline Middle & $13(36.1)$ & $62(55.9)$ & \\
\hline Low & $17(47.2)$ & $34(30.6)$ & \\
\hline BDI-Il total score & $7.27 \pm 8.29$ & $3.59 \pm 5.37$ & $0.014^{*}$ \\
\hline
\end{tabular}

Data are presented as $\mathrm{n}(\%)$ or mean \pm standard deviation. ${ }^{*} \mathrm{p}<0.05$. BDI-II: Beck Depression Inventory-II, CES-DC: Center for Epidemiological Studies Depression Scale for Children, SCARED: Screen for Children Anxiety Emotional Disorders, ACE: adverse childhood experience 
Table 3. Comparison of offspring's traumatic experiences and PTSD according to maternal ACES

\begin{tabular}{lccc}
\hline & Maternal ACEs $(+)(\mathrm{n}=37)$ & Maternal ACEs $(-)(\mathrm{n}=119)$ & $\mathrm{p}$ \\
\hline Offspring's ETISR-SF & & & $<0.001^{*}$ \\
General trauma, $\geq 1$ & $21(56.8)$ & $23(19.3)$ & $<0.001^{*}$ \\
Physical trauma, $\geq 1$ & $16(43.2)$ & $13(10.9)$ & 0.003 \\
Emotional trauma, $\geq 1$ & $9(24.3)$ & $8(6.7)$ & 0.072 \\
Sexual trauma, $\geq 1$ & $1(2.7)$ & $0(0)$ & $<0.001^{*}$ \\
Total ETISR-SF score & $2.78 \pm 3.63$ & $0.55 \pm 1.30$ & $<0.001^{*}$ \\
Offspring's PTSD & $19(51.4)$ & $15(12.6)$ & $<$
\end{tabular}

Data are presented as $n(\%)$ or mean \pm standard deviation. ${ }^{*} p<0.01$. ACE: adverse childhood experience, ETISR-SF: Early Trauma Inventory Self-Report-Short Form, PTSD: post-traumatic stress disorder

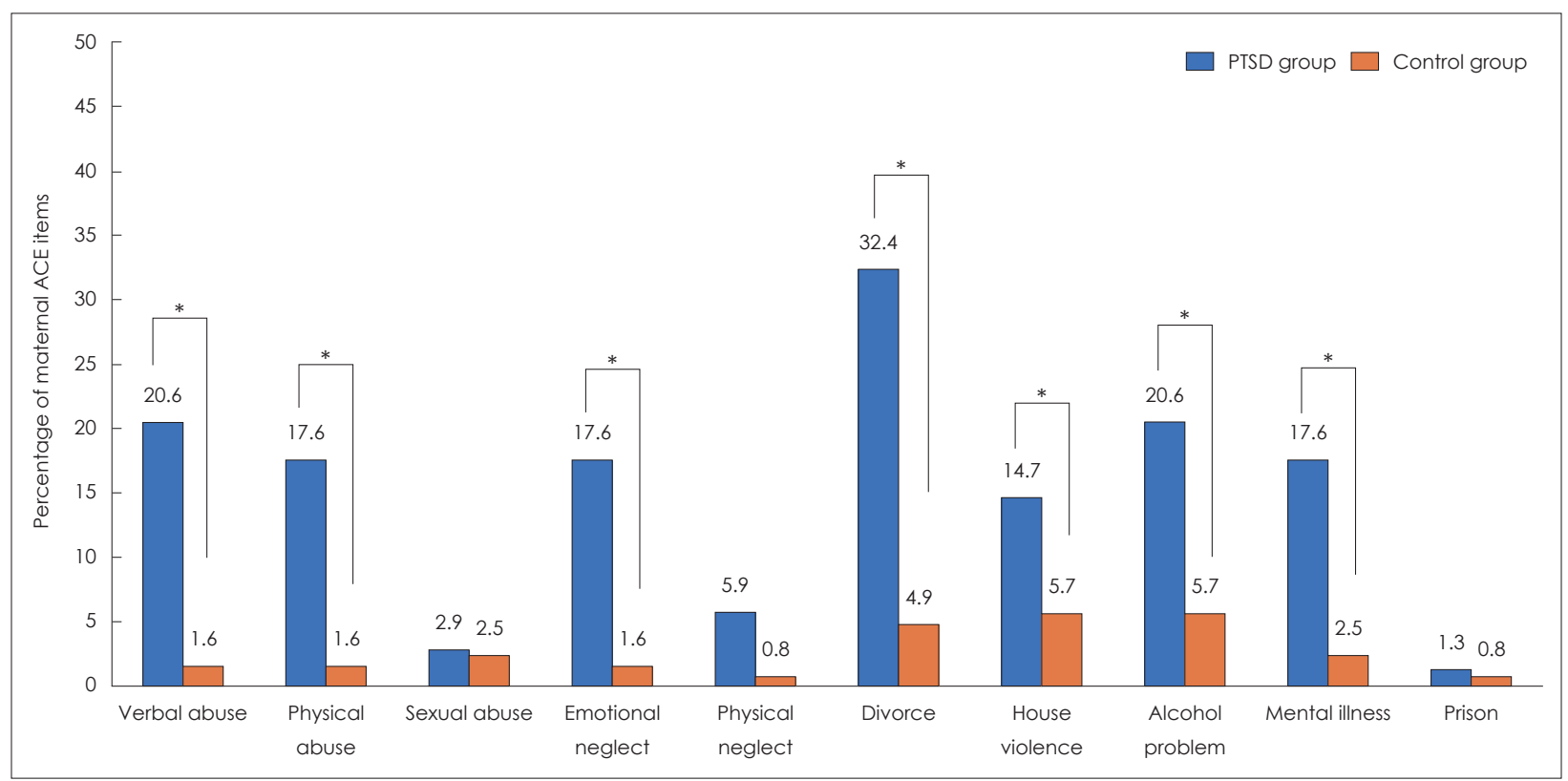

Fig. 2. Comparison of frequency of maternal ACE item between offspring's PTSD group and control group. ${ }^{*} p<0.001$. ACE: adverse childhood experiences, PTSD: post-traumatic stress disorder.

Table 4. Multivariable logistic regression for the offspring's post-traumatic stress disorder by maternal ACE domains

\begin{tabular}{|c|c|c|c|c|c|}
\hline & B & SE & OR & $95 \% \mathrm{Cl}$ & $\mathrm{p}$ \\
\hline Offspring's ETISR-SF total score & 0.429 & 0.158 & 1.536 & $1.127-2.904$ & $0.007^{\dagger}$ \\
\hline Maternal BDI-II total score & 0.103 & 0.047 & 1.109 & $1.012-1.215$ & $0.027^{*}$ \\
\hline \multicolumn{6}{|l|}{ Maternal ACE domain } \\
\hline Child abuse & 0.060 & 0.498 & 1.062 & $0.400-2.821$ & 0.903 \\
\hline Child neglect & 1.524 & 1.157 & 4.589 & $0.475-44.339$ & 0.188 \\
\hline Household dysfunction & 1.101 & 0.453 & 3.008 & $1.238-7.305$ & $0.05^{\dagger}$ \\
\hline
\end{tabular}

Adjusted socioeconomic status, maternal age, maternal education level, offspring's age and sex, total score of Center for Epidemiological Studies Depression Scale for Children and Screen for Children Anxiety-Related Disorders. Hosmer-Lemeshow goodness of fit $(p=0.153) .{ }^{*} p<0.05,{ }^{\dagger} p<0.01$. ACE: adverse childhood experience, B: regression coefficient, BDI-II: Beck Depression Inventory-II, Cl: confidence interval, ETISR-SF: Early Trauma Inventory Self-Report-Short Form, OR: odds ratio, SE: standard error of B

and more than three maternal ACEs were significantly associated with PTSD in the offspring.

Our results are consistent with those of previous studies, namely that parents' traumatic experiences in childhood increased the risk of psychiatric disorders in their offspring. Haynes et al. [21]. reported that when the offspring had de- pression and anxiety, the caregivers had many ACEs, such as alcohol use, domestic violence, mental health problems, and verbal abuse. There are few studies on the relationship between maternal ACEs and PTSD in the offspring. Maternal childhood physical and sexual abuse and history of multiple ACEs are significant predictors of PTSD in the offspring [22]. 
Table 5. Multivariable logistic regression for the offspring's post-traumatic stress disorder by maternal ACE level

\begin{tabular}{|c|c|c|c|c|c|}
\hline & B & SE & OR & $95 \% \mathrm{Cl}$ & $\mathrm{p}$ \\
\hline Offspring's ETISR-SF total score & 0.399 & 0.147 & 1.490 & $1.118-1.986$ & $0.007^{\dagger}$ \\
\hline Maternal BDI-II total score & 0.106 & 0.046 & 1.111 & $1.016-1.216$ & $0.021^{*}$ \\
\hline \multicolumn{6}{|l|}{ Maternal total ACE score } \\
\hline 0 & \multicolumn{3}{|c|}{ Reference } & & \\
\hline 1 & 1.390 & 0.714 & 4.013 & $0.990-16.265$ & 0.052 \\
\hline 2 & 1.553 & 1.014 & 4.724 & $0.647-34.491$ & 0.126 \\
\hline$\geq 3$ & 2.362 & 1.057 & 10.613 & $1.338-84.172$ & $0.025^{*}$ \\
\hline
\end{tabular}

Adjusted socioeconomic status, maternal age, maternal education level, offspring's age and sex, total score of Center for Epidemiological Studies Depression Scale for Children and Screen for Children Anxiety-Related Disorders. Hosmer-Lemeshow goodness of fit $(p=0.416) .{ }^{*} p<0.05,{ }^{\dagger} p<0.01$. ACE: adverse childhood experience, B: regression coefficient, BDI-II: Beck Depression Inventory-II, Cl: confidence interval, ETISR-SF: Early Trauma Inventory Self-Report-Short Form, OR: odds ratio, SE: standard error of B

Previous studies have shown that if parents have PTSD, their offspring are more likely to suffer from childhood trauma [23], and maternal chronic PTSD significantly predicts PTSD symptoms in the offspring [24].

Our finding that mothers who experienced $\geq 3$ ACEs showed a higher ORs of PTSD in the offspring suggests that maternal ACEs above a certain threshold can affect intergenerational transmission of PTSD to the offspring. A previous study reported that when the caregiver is exposed to four or more ACEs, the offspring are more likely to experience anxiety and depression, suggesting a dose-response-based transmission of ACEs [21].

In the present study, maternal household dysfunction was a risk factor associated with PTSD in the offspring. Maternal household dysfunction, such as parental alcohol problems, psychiatric disorders, and divorce, increased the risk of poor health behaviors and symptoms in the mothers, such as alcoholism, depression, and suicide ideation [8]. Maternal childhood adversity affects the offspring's mental health through emotional neglect or abuse. In previous studies, parental alcohol use disorder was related to physical, sexual, and witness violence experienced by the children and was a risk factor for lifetime PTSD [25].

Regarding the mechanism of intergenerational transmission leading to maternal ACEs and offspring's traumatic experience and PTSD, there is a possibility that the offspring's biological vulnerability that leads to trauma-related mood and anxiety symptoms after trauma exposure is increased. ACEs cause changes in hypothalamic-pituitary-adrenal (HPA) axis-relataed gene expression via epigenetic regulation, which affects the dysregulation of the glucocorticoid signaling and stress neurocircuitry [26], and the offspring can be vulnerable to mood and anxiety disorders, including PTSD. Second, maternal ACEs are likely to lead to psychiatric disorders such as maternal depression [27]. Traumatic experiences such as child abuse in the family or maternal mental health problems make it difficult for the mothers to help their offspring regulate their emotions after trauma. In a previous study, the mother's ex- periences of domestic violence and related PTSD symptoms were reported as predictors of poor emotional and behavioral outcomes when their children experienced adverse life events [28]. In addition, maternal ACEs tend to lead to dysfunctional parenting of the offspring, resulting in difficulties related to emotional and arousal regulation in the offspring. Third, maternal ACEs increase the risk of traumatic experiences in the offspring. The mother's childhood maltreatment directly predicts offspring victimization [29]. Higher parental ACEs predict higher ACEs in the offspring [30].

This study has several limitations. First, it is difficult to generalize the results because the present study was conducted in Jeju area. Second, although we used DPS including the DSM-IV criteria to confirm PTSD, DPS is not a structured interview tool. Third, since this study evaluated the DPS data reported by the mothers, there is a possibility of the reports being different from the real experience of the offspring. Fourth, there is a possibility of recall bias or underestimation of ACEs and traumatic experiences because the present study used a retrospective questionnaire to evaluate maternal ACEs and traumatic experiences in the offspring. Fifth, this was a crosssectional study. In the future, prospective studies on intergenerational transmission of ACE are needed.

The present study suggests intergenerational transmission through which maternal ACEs significantly affect the occurrence of PTSD in the offspring. Future studies should investigate the mediating factors affecting PTSD in the offspring, such as psychiatric disorders in the mother and offspring, parenting attitude, and temperament of the offspring.

\section{CONCLUSION}

This study showed that maternal ACEs were associated with traumatic experiences and PTSD in the offspring through intergenerational transmission. Higher maternal ACE scores and experience of household dysfunction are risk factors for psychiatric disorders in the offspring. Maternal ACEs are an 
important issue in the research on children's mental health problems and intergenerational transmission of ACEs. Further research is needed to determine the underlying mechanisms of intergenerational transmission of ACE and psychopathology in the offspring.

\section{Acknowledgments}

This work was supported by a research grant from Jeju National University Hospital in 2018.

\section{Conflicts of Interest}

The authors have no potential conflicts of interest to disclose.

\section{Author Contributions}

Conceptualization: Na Ri Kang, Bung-Nyun Kim. Formal analysis: Min Chull Na, Na Ri Kang. Investigation: Bung-Nyun Kim, Duk-Soo Moon. Methodology: all authors. Supervision: Moon Doo Kim, Joon Hyuk Park, Young-Eun Jung. Writing-original draft: Min Chull Na. Writing—review \& editing: Duk-Soo Moon, Na Ri Kang.

\section{ORCID iDs}

$\begin{array}{ll}\text { Min Chull Na } & \text { https://orcid.org/0000-0003-1991-5545 } \\ \text { Moon Doo Kim } & \text { https://orcid.org/0000-0002-6441-630X } \\ \text { Joon Hyuk Park } & \text { https://orcid.org/0000-0002-0396-5284 } \\ \text { Young-Eun Jung } & \text { https://orcid.org/0000-0001-7608-0009 } \\ \text { Duk-Soo Moon } & \text { https://orcid.org/0000-0001-7878-3410 } \\ \text { Hyun-Ju Yang } & \text { https://orcid.org/0000-0003-0027-2834 } \\ \text { Bung-Nyun Kim } & \text { https://orcid.org/0000-0002-2403-3291 } \\ \text { Na Ri Kang } & \text { https://orcid.org/0000-0002-2086-0927 }\end{array}$

\section{REFERENCES}

1) Felitti VJ, Anda RF, Nordenberg D, Williamson DF, Spitz AM, Edwards V, et al. Relationship of childhood abuse and household dysfunction to many of the leading causes of death in adults: the Adverse Childhood Experiences (ACE) Study. Am J Prev Med 1998;14:245-258

2) Björkenstam E, Burström B, Vinnerljung B, Kosidou K. Childhood adversity and psychiatric disorder in young adulthood: an analysis of 107,704 Swedes. J Psychiatr Res 2016;77:67-75.

3) Lee H, Kim Y, Terry J. Adverse childhood experiences (ACEs) on mental disorders in young adulthood: latent classes and community violence exposure. Prev Med 2020;134:106039.

4) Plant DT, Pawlby S, Pariante CM, Jones FW. When one childhood meets another - maternal childhood trauma and offspring child psychopathology: a systematic review. Clin Child Psychol Psychiatry 2018;23:483-500.

5) Roberts AL, Chen Y, Slopen N, McLaughlin KA, Koenen KC, Austin SB. Maternal experience of abuse in childhood and depressive symptoms in adolescent and adult offspring: a 21-year longitudinal study. Depress Anxiety 2015;32:709-719.

6) Schickedanz A, Halfon N, Sastry N, Chung PJ. Parents' adverse childhood experiences and their children's behavioral health problems. Pediatrics 2018;142:e20180023.

7) Negriff S, Palmer Molina A, Hackman DA. Parental exposure to childhood maltreatment and offspring's mental health: investigating pathways through parental adversity and offspring exposure to maltreatment. Child Maltreat 2020;25:422-432.

8) Felitti VJ, Anda RF, Nordenberg D, Williamson DF, Spitz AM, Edwards V, et al. Reprint of: relationship of childhood abuse and household dysfunction to many of the leading causes of death in adults: the Adverse Childhood Experiences (ACE) Study. Am J Prev Med 2019;56:774-786.

9) Bremner JD, Bolus R, Mayer EA. Psychometric properties of the early trauma inventory-self report. J Nerv Ment Dis 2007;195:211218 .

10) Jeon JR, Lee EH, Lee SW, Jeong EG, Kim JH, Lee D, et al. The early trauma inventory self report-short form: psychometric properties of the Korean version. Psychiatry Investig 2012;9:229-235.

11) Shaffer D, Fisher P, Lucas CP, Dulcan MK, Schwab-Stone ME. NIMH diagnostic Interview schedule for children version IV (NIMH DISC-IV): description, differences from previous versions, and reliability of some common diagnoses. J Am Acad Child Adolesc Psychiatry 2000;39:28-38.

12) Leung PW, Lucas CP, Hung SF, Kwong SL, Tang CP, Lee CC, et al. The test-retest reliability and screening efficiency of DISC predictive scales-version 4.32 (DPS-4.32) with Chinese children/youths. Eur Child Adolesc Psychiatry 2005;14:461-465.

13) Cho SC, Go BJ, Kim BS, Kim BN, Kim JW, Shin MS, et al. The 2005 Seoul child and adolescent mental health survey. Seoul: Child and Adolescent Mental Health Center;2006.

14) Cubo E, Velasco SS, Benito VD, Villaverde VA, Galín JM, Santidrián AM, et al. Psychometric Attributes of the DISC predictive scales. Clin Pract Epidemiol Ment Health 2010;6:86-93.

15) Fendrich M, Weissman MM, Warner V. Screening for depressive disorder in children and adolescents: validating the Center for Epidemiologic Studies Depression Scale for Children. Am J Epidemiol 1990;131:538-551.

16) Shin SC, Kim MK, Yun KS, Kim JH, Lee MS, Moon SJ, et al. The center for epidemiologic studies-depression scale: its use in Korea. J Korean Neuropsychiatr Assoc 1991;30:752-767.

17) Birmaher B, Brent DA, Chiappetta L, Bridge J, Monga S, Baugher M. Psychometric properties of the Screen for Child Anxiety Related Emotional Disorders (SCARED): a replication study. J Am Acad Child Adolesc Psychiatry 1999;38:1230-1236.

18) Kim M. A validation study of the SCARED: for the elementay school uppergrades and middle school students [dissertation]. Seoul: Sookmyung Women's University Graduate School;2010.

19) Beck AT, Steer RA, Brown GK. Manual for the Beck Depression Inventory-II. San Antonio, TX: Psychological Corporation;1996. p.490-498.

20) Sung HM, Kim JB, Park YN, Bai DS, Lee SH, Ahn HN. A study on the reliability and the validity of Korean version of the Beck Depression Inventory-II (BDI-II). J Korean Soc Biol Ther Psychiatry 2008;14:201-212.

21) Haynes E, Crouch E, Probst J, Radcliff E, Bennett K, Glover S. Exploring the association between a parent's exposure to Adverse Childhood Experiences (ACEs) and outcomes of depression and anxiety among their children. Child Youth Serv Rev 2020;113: 105013.

22) Karatzias T, Shevlin M, Fyvie C, Grandison G, Garozi M, Latham E, et al. Adverse and benevolent childhood experiences in posttraumatic stress disorder (PTSD) and complex PTSD (CPTSD): implications for trauma-focused therapies. Eur J Psychotraumatol 2020;11:1793599.

23) Yehuda R, Halligan SL, Grossman R. Childhood trauma and risk for PTSD: relationship to intergenerational effects of trauma, parental PTSD, and cortisol excretion. Dev Psychopathol 2001;13: 733-753.

24) Chen XY, Chen J, Shi X, Jiang M, Li Y, Zhou Y, et al. Trajectories of maternal symptoms of posttraumatic stress disorder predict longterm mental health of children following the Wenchuan earthquake in China: a 10-year follow-up study. J Affect Disord 2020;266: 201-206.

25) Bender AK, Bucholz KK, Edenberg HJ, Kramer JR, Anokhin AP, Meyers JL, et al. Trauma exposure and post-traumatic stress disor- 
der among youth in a high-risk family study: associations with maternal and paternal alcohol use disorder. J Fam Trauma Child Custody Child Dev 2020;17:116-134.

26) McGowan PO. Epigenomic mechanisms of early adversity and HPA dysfunction: considerations for PTSD research. Front Psychiatrya 2013;4:110.

27) Chapman DP, Whitfield CL, Felitti VJ, Dube SR, Edwards VJ, Anda RF. Adverse childhood experiences and the risk of depressive disorders in adulthood. J Affect Disord 2004;82:217-225.

28) Yehuda R, Halligan SL, Bierer LM. Relationship of parental trauma exposure and PTSD to PTSD, depressive and anxiety disor- ders in offspring. J Psychiatr Res 2001;35:261-270.

29) Madigan S, Cyr C, Eirich R, Fearon RMP, Ly A, Rash C, et al. Testing the cycle of maltreatment hypothesis: meta-analytic evidence of the intergenerational transmission of child maltreatment. Dev Psychopathol 2019;31:23-51.

30) Narayan AJ, Kalstabakken AW, Labella MH, Nerenberg LS, Monn AR, Masten AS. Intergenerational continuity of adverse childhood experiences in homeless families: unpacking exposure to maltreatment versus family dysfunction. Am J Orthopsychiatry 2017; 87:3-14. 